Acta Crystallographica Section D

Biological

Crystallography

ISSN 0907-4449

Ricardo M. Neto-Silva, ${ }^{a}$ Sandra Macedo-Ribeiro, ${ }^{\mathrm{b}, \mathrm{c}}$ Pedro J. B. Pereira, ${ }^{\mathrm{a}}$ M. Coll, ${ }^{\mathrm{c}}$ Maria J. Saraiva $^{\mathrm{d}, \mathrm{e}}$ and Ana M. Damas ${ }^{\mathrm{a}, \mathrm{e}}$ *

aMolecular Structure Group, Institute for Molecular and Cell Biology, Rua do Campo Alegre No. 823, 4150 Porto, Portugal, ' ${ }^{\mathbf{b}}$ Center for Neurosciences and Cell Biology, IBILI, 3rd Floor, Azinhaga de Sta Comba, Celas. 3000-354 Coimbra, Portugal, ' Instituto de Biologia Molecular de Barcelona, CSIC, Parc Científic de Barcelona, Josep Samitier 1-5, 08028 Barcelona, Spain, ${ }^{\mathbf{d}}$ Molecular Neurobiology Group, Institute for Molecular and Cell Biology, Rua do Campo Alegre No. 823, 4150 Porto, Portugal, and ${ }^{\mathbf{e}}$ ICBAS - Instituto de Ciências Biomédicas Abel Salazar, University of Porto, Largo Prof. Abel Salazar No. 2, 4099-003 Porto, Portugal

Correspondence e-mail: amdamas@ibmc.up.pt

\title{
X-ray crystallographic studies of two transthyretin variants: further insights into amyloidogenesis
}

Transthyretin (TTR) is a homotetrameric plasma protein that, as a result of a set of not yet fully characterized conformational changes, forms fibrillar aggregates that are the major protein component of amyloid deposits. More than 80 mutations associated with TTR amyloid deposition have been described in the literature. X-ray crystallography was used to elucidate the three-dimensional structure of two important TTR variants: TTR Y78F, an amyloidogenic protein, and TTR $\mathrm{R} 104 \mathrm{H}$, which is associated with a protective effect over the amyloidogenic V30M mutation. The structures of those two TTR variants have been determined in space group $P 2_{1} 2_{1} 2$ to 1.55 and $1.60 \AA$ resolution, respectively, using molecularreplacement techniques. Detailed analysis of the protein model for TTR Y78F indicates a destabilization of the contacts between the $\alpha$-helix and $A B$ loop and the body of the molecule, intimately related to the amyloidogenic nature; contrastingly, in the TTR R104H variant new contacts involving the $\mathrm{N}$-terminal region and His104 are clearly antagonists of amyloid formation.

\section{Introduction}

Transthyretin (TTR) is a protein found both in the plasma and in the cerebrospinal fluid that is known to bind thyroxine (L-T4) and retinol-binding protein (RBP). Its relevant biological form is a homotetramer in which each subunit is composed of 127 amino acids. The three-dimensional structure of TTR, as determined by X-ray crystallography (Blake et al., 1978), shows that each monomer is composed of eight $\beta$-strands $(A-H)$ that assemble non-covalently in a $\beta$-sandwich, with strands $C B E F$ facing strands $D A G H$ (Fig. 1). The single $\alpha$-helical element that is present in each monomer comprises a short segment of seven amino acids located between strands $E$ and $F$. The dimer, which is the repeating unit in the crystallographic asymmetric unit of the wild-type protein and several variants, is composed of one pair of eightstranded $\beta$-sandwiches, which are held together through hydrogen bonds between the strands $H H^{\prime}$ and $F F^{\prime}$. The tetramer in the crystallographic structure is formed when two dimers related by a twofold axis associate through contacts between the $A B$ loops of one dimer and the $H$ strands of the other dimer.

Structures of TTR in complex with thyroxine (T4) and RBP have also been determined by X-ray crystallography (Wojtczak, 1996; Monaco et al., 1995). The extracellular deposition of transthyretin, in the form of fibrillar aggregates characterized by an extensive $\beta$-sheet structure, has been shown to be associated with senile systemic amyloidosis (SSA) or, more commonly, with familial amyloidotic polyneuropathy (FAP). In the former, the wild-type (WT) protein is the major
Received 5 November 2004 Accepted 23 December 2004

PDB References: TTR R104H, $1 \mathrm{x} 7 \mathrm{t}, \mathrm{r} 1 \mathrm{x} 7 \mathrm{tsf}$; TTR Y78F, $1 \times 7 \mathrm{~s}$, $\mathrm{r} 1 \times 7 \mathrm{ssf}$. 
component of the aggregates, whereas in the latter a variant protein is present (Saraiva, 1996). Thus far, over 80 amyloidogenic and non-amyloidogenic mutations have been found of TTR (Saraiva, 2001). There are only a restricted number of nonpathogenic mutations, some of which have been shown to exert a protective effect on heterozygotic individual carriers of an amyloidogenic TTR variant.

In the case of TTR Y78F, the mutation is located in the single $\alpha$-helix present in each monomer. This variant was engineered after careful analysis of the structure of the TTR L55P mutant, which is one of the most aggressive variants described so far and has been reported as being implicated in the early onset of FAP (Jacobson et al., 1992). The threedimensional structure of TTR suggested that hydrogen bonds between the $\mathrm{OH}$ group of Tyr78 and some residues belonging to the $A B$ loop play a key role in the overall fold and stability of the protein (Sebastião et al., 1998). In fact, biochemical studies have shown that TTR Y78F is highly prone to fibril formation in vitro upon acidification (Redondo et al., 2000). Furthermore, and most interestingly, this variant has been shown to react in its soluble tetrameric form with a monoclonal antibody reported to only recognize structures presenting a highly amyloidogenic nature (Goldsteins et al., 1999). It is possible that this variant resembles an early intermediate in the fibrillogenesis cascade (Redondo et al., 2000). Recently, the clinical relevance of the mutation was shown by its identification in families of Italian origin; it has been reported to be causative of peripheral neuropathy, carpal tunnel syndrome, skin amyloidosis (Magy et al., 2003) and also cardiomyopathy (Anesi et al., 2001).

Only a few of the various mutations of TTR identified to date display non-amyloidogenic effects. Of those, a more restricted group has been associated with a protective effect against the development of the disease in heterozygotic compound carriers of an amyloidogenic mutation. This is the case for the mutation involving an arginine to histidine exchange at position 104 of the TTR amino-acid sequence, which is located in the protein $F G$ loop, and that was originally identified in Japanese heterozygous individuals suffering from FAP (Terazaki et al., 1999). Individuals carrying this mutation show increased levels of TTR and RBP in the serum, which is in accordance with previous observations for carriers of TTR $\mathrm{T} 119 \mathrm{M}$, another mutation conferring a protective effect in carriers of amyloidogenic TTR V30M. Likewise, TTR V30M/ $\mathrm{R} 104 \mathrm{H}$ does not dissociate in its monomeric subunits as easily as the V30M protein, but does however show a T4-binding affinity that is lower than normal (Almeida et al., 2000).

The crystal structures of several TTR variants have consistently revealed, with the exceptions of L55P (Sebastião et al., 1998) and the G53S/E54D/L55S triple mutant (Eneqvist et al., 2000), that there are no major structural changes induced upon mutation.

Given the rather poor knowledge of the structure of TTRamyloid fibrils and the difficulties encountered in obtaining a model with atomic resolution, an integrated analysis of the structural relevant features of both amyloidogenic and nonamyloidogenic/protective TTR variants should prove crucial to shed some light on the pathway leading to fibrillogenesis. Using X-ray crystallography, we have solved the structures of two variants with opposite clinical outcomes: TTR R104H, a protective mutation with increased T4-binding affinity, and TTR Y78F, a highly amyloidogenic mutation with a fold in its soluble form that might represent an early intermediate in the fibrillogenesis pathway.

\section{Material and methods}

\subsection{Protein preparation and crystallization}

Recombinant TTR R104H and TTR Y78F were isolated and purified as described elsewhere (Almeida et al., 2000; Redondo et al., 2000). The proteins were crystallized by vapour-diffusion methods after diluting the protein solutions $\left(10 \mathrm{mg} \mathrm{ml}^{-1}\right)$ in a $1: 1$ ratio with the reservoir solutions. Crystals of the recombinant TTR R104H (10 mg ml$\left.{ }^{-1}\right)$ were grown at $293 \mathrm{~K}$ using a reservoir solution consisting of $18 \% \mathrm{PEG}$ 3350, $0.2 \mathrm{M}$ ammonium sulfate and $50 \mathrm{~m} M$ sodium acetate $\mathrm{pH}$ 5.1. The Y78F TTR variant was crystallized at $287 \mathrm{~K}$ with a reservoir solution consisting of $2.0 \mathrm{M}$ ammonium sulfate, $7 \%$ glycerol and $0.2 \mathrm{M}$ sodium acetate $\mathrm{pH} 4.8$.

Crystals were sequentially transferred to stabilizing solutions (27\% PEG 3350, $0.3 M$ ammonium sulfate, $0.75 M$ sodium acetate $\mathrm{pH} 5.1$ for $\mathrm{R} 104 \mathrm{H}$ and $2.5 M$ ammonium

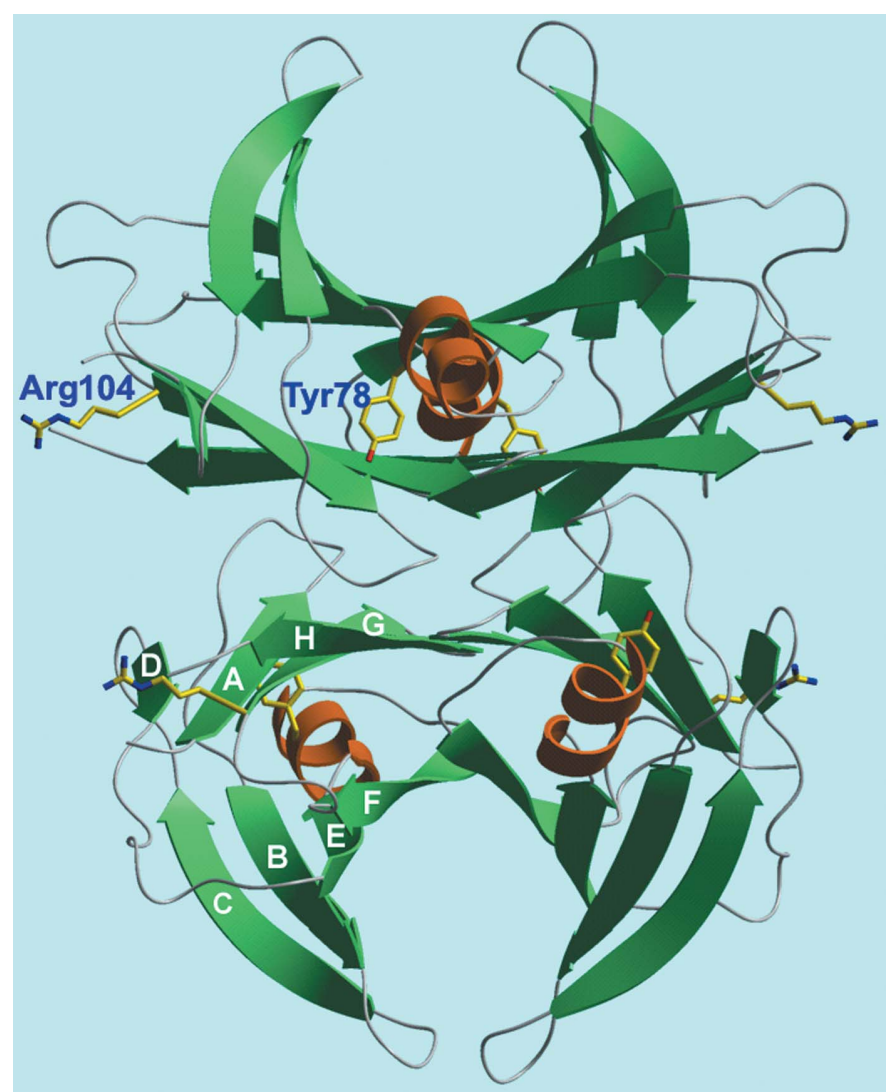

Figure 1

Schematic representation of the crystallographic TTR tetramer showing the location of the amino-acid substitutions (Y78F and R104H) for each of the studied variants. The $\beta$-strands of one of the monomers are labelled $A-H$. 
sulfate, $0.2 \mathrm{M}$ sodium acetate $\mathrm{pH} 4.8$ for $\mathrm{Y} 78 \mathrm{~F}$ ) containing increasing concentrations of glycerol $(5-25 \%)$ and finally cryocooled in liquid nitrogen.

\subsection{Data collection and processing}

X-ray diffraction data were collected at $100 \mathrm{~K}$ using synchrotron radiation on beamline ID14-1 at the European Synchrotron Radiation Facility (ESRF) in Grenoble. Diffraction data for both variants were collected on an ADSC Q4R CCD detector from a single crystal (both variants) at a wavelength of $0.934 \AA$. Data were evaluated and processed with MOSFLM (Leslie, 1992) and reduced with SCALA and TRUNCATE (Collaborative Computational Project, Number 4 , 1994). Crystals of recombinant TTR R104H and TTR Y78F diffract to a maximum resolution of 1.54 and $1.55 \AA$, respectively, and both variants belong to space group $P 2_{1} 22_{1} 2$. The

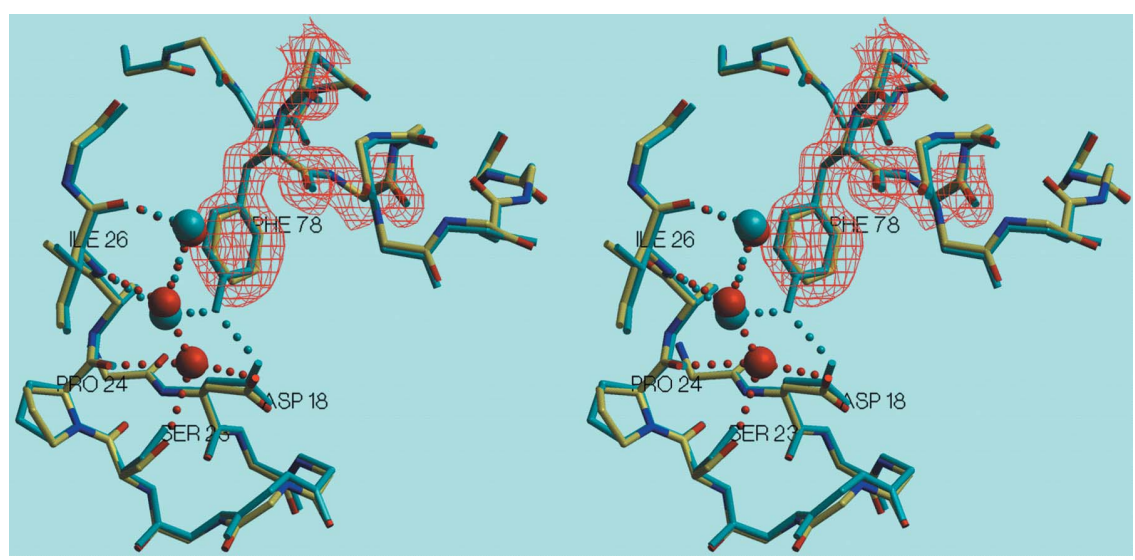

Figure 2

A stereoview of a superposition between the WT (in blue) and TTR Y78F (colour-coded with $\mathrm{O}$ atoms in red, $\mathrm{N}$ atoms in blue and $\mathrm{C}$ atoms in yellow) in the region of the mutation. In WT TTR, the hydroxyl group of Tyr78 establishes key contacts with residues in the $A B$ loop, providing a relevant association between two regions located far apart in the protein primary structure. In the Tyr78Phe variant, contacts between the $A B$ loop and the protein core are lost. An additional water molecule ensures near-native hydrogen bonds within the $A B$-loop region, preventing its complete displacement. The final $\left(2 F_{\mathrm{o}}-F_{\mathrm{c}}\right)$ electron-density map (red, contoured at the $1 \sigma$ level) is shown around Phe78.

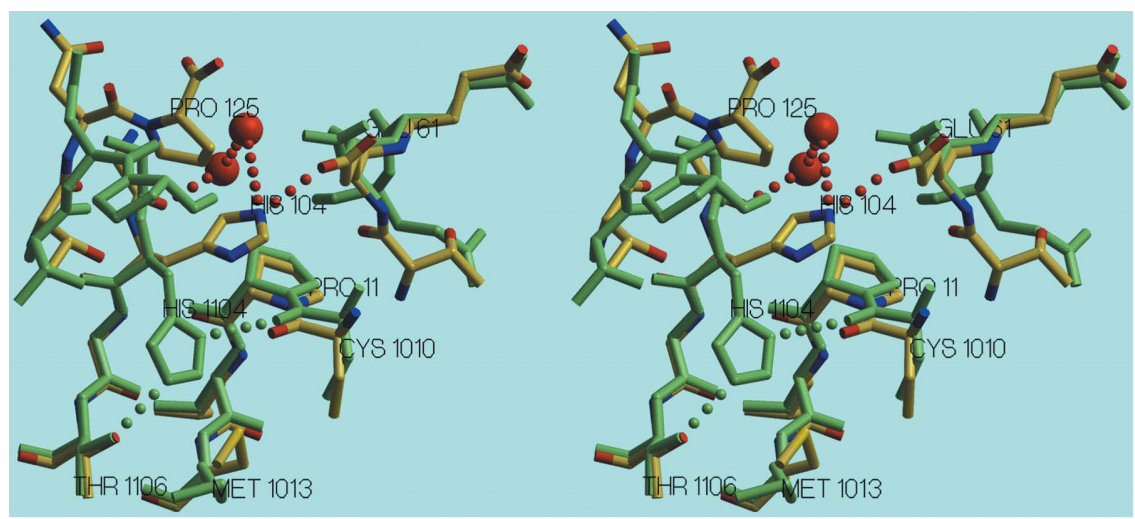

Figure 3

A stereoview of the superposition between monomers $A$ (colour coded with $\mathrm{O}$ atoms in red, $\mathrm{N}$ atoms in blue and $\mathrm{C}$ atoms in yellow) and $B$ (green) of R104H TTR around the mutated residue. N- and C-terminal stabilization is observed in monomer $A$ (numbered 10-125), where the mutated residue is buried between Pro11 and Pro125. In monomer $B$ (numbered 1010$1125)$ the imidazole ring establishes a hydrogen bond with the Cys10 main-chain carbonyl. unit-cell parameters are $a=42.72, b=86.08, c=63.80 \AA$, $\alpha=\beta=\gamma=90^{\circ}$ for TTR Y78F and $a=43.03, b=85.52$, $c=64.24 \AA, \alpha=\beta=\gamma=90^{\circ}$ for TTR R104H. The Matthews coefficient is $2.1 \AA^{3} \mathrm{Da}^{-1}$ for both structures.

\subsection{Structure determination and refinement}

The structures were solved by molecular-replacement techniques using AMoRe (Navaza, 1994) and as a search model the coordinates of wild-type TTR (Steinrauf et al., 1993; PDB code 1tta) for R104H TTR and the T119M variant mutated amino acids were replaced by Ala residues in both search models. Calculations were performed with data in the 15-3.5 A resolution range and both the rotation and translation functions produced correct solutions that were further improved with a final rigid body refinement. An $R$ factor of 0.360 and a correlation coefficient of 0.692 were found for the TTR Y78F variant, whereas in the case of TTR R104H the values were 0.367 and 0.616 , respectively.

Model building was performed on an SGI Graphic Workstation using the program TURBO-FRODO (Roussel \& Cambillau, 1989). Positional and individual temperature-factor refinement was carried out with CNS (Brünger et al., 1998) for both TTR variants. The computed $\sigma_{A}$-weighted $\left(2 F_{\text {obs }}-F_{\text {calc }}\right)$ and $\left(F_{\text {obs }}-F_{\text {calc }}\right)$-type electron-density maps showed the expected positive difference density for the new amino-acid residues introduced by the mutation in each variant. Automated refinement was alternated with manual fitting of the model to the electron density. For each round of refinement, the positions of the crystallographic refinement generated water molecules were checked manually. The final isotropically refined models of both variants were further refined with SHELX97-2 (Sheldrick \& Schneider, 1997) in two rounds of 20 conjugate-gradient least-squares cycles using isotropic thermal parameters. Structure validation was performed with the programs PROCHECK (Laskowski et al., 1993) and WHAT_CHECK (Hooft et al., 1996). Dataprocessing and refinement statistics are outlined in Table 1 . The $\mathrm{N}$-terminal residues 1-8 are not well defined in the electron-density maps and are thus missing in the final structures of both TTR variants. Additionally, residue 9 is not well defined in monomer $B$ of TTR R104H and in both subunits of the TTR Y78F model. Likewise, the C-terminal residues were excluded from both models owing to their weak electron 
Table 1

Data-collection and refinement statistics.

Values in parentheses are for the last shell.

\begin{tabular}{lll}
\hline & TTR Y78F & TTR R104H \\
\hline $\begin{array}{ll}\text { Data collection } \\
\text { Space group }\end{array}$ & $P 2{ }_{1} 2$ & \\
Unit-cell parameters & & $P 2{ }_{1} 2$ \\
$\quad a(\AA)$ & 42.72 & 43.03 \\
$\quad b(\AA)$ & 86.08 & 85.52 \\
$\quad c(\AA)$ & 63.80 & 64.24 \\
$\alpha=\beta=\gamma\left({ }^{\circ}\right)$ & 90 & 90 \\
Resolution $(\AA)$ & 1.55 & 1.54 \\
No. of observations (total/unique) & $295939 / 33669$ & $314508 / 36082$ \\
$R_{\text {merge }} \dagger(\%)$ & $6.4(31.3)$ & $9.7(28.1)$ \\
Completeness $(\%)$ & $98.0(96.7)$ & $91.8(73.7)$ \\
$I / \sigma(I)$ & $12.3(5.7)$ & $14.2(4.9)$ \\
Structure refinement & & \\
Resolution range & $10.0-1.55$ & $10.0-1.60$ \\
$R_{\text {cryst }} \ddagger / R_{\text {free }}(\%)$ & $19.4 / 23.8$ & $19.9 / 25.2$ \\
No. of reflections (working/free) & $31928 / 1711$ & $29601 / 1553$ \\
No. of water molecules & 196 & 187 \\
Residues in alternate conformations & & Cys10A, Ser117B \\
Average $B$ factor $\left(\AA^{2}\right)$ & 23.69 & 26.62 \\
R.m.s. bonded $B$ s $\left(\AA^{2}\right)$ & 4.625 & 5.05 \\
R.m.s. deviations from standard geometry & & \\
$\quad$ Bond lengths $(\AA)$ & 0.009 & 0.008 \\
$\quad$ Bond angles $\left({ }^{\circ}\right)$ & 2.147 & 2.076 \\
Ramachandran plot & & \\
Core $(\%)$ & 92.0 & 90.3 \\
Allowed $(\%)$ & 8.0 & 9.7 \\
\hline
\end{tabular}

$\dagger R_{\text {merge }}=\sum \sum\left|I(h)_{i}-\langle I(h)\rangle\right| / \sum \sum I(h)$, where $I(h)_{i}$ is the observed intensity of the $i$ th observation of reflection $h$ and $\langle I(h)\rangle$ is the mean intensity of reflection $h$ over all measurements of $I(h) . \quad \ddagger R_{\text {cryst }}=\sum\left(F_{\mathrm{o}}-F_{\mathrm{c}}\right) / \sum F_{\mathrm{c}} . \quad \S R_{\text {free }}$ is the cross-validation $R$ factor computed for the test set of reflections ( $5 \%$ of the total number of reflections) that were omitted in the refinement process.

densities. These include Lys126 and Glu127 in monomer $A$ of the R104H variant, and Pro125, Lys126 and Glu127 in monomer $B$ of the R104H variant, and in both monomers of TTR Y78F. Residues Cys10 and Ser117 are found in two alternate conformations in monomers $A$ and $B$, respectively, of TTR R104H.

\section{Results and discussion}

\subsection{TTR Y78F}

TTR Y78F crystallizes isomorphously with the WT protein in space group $P 22_{1} 2$, with two monomers in the asymmetric unit, from a solution containing predominantly the tetrameric form of the protein. Diffraction data were collected to $1.55 \AA$ resolution using synchrotron radiation. Each TTR monomeric subunit is composed of 127 amino acids that could be consistently modelled in both monomers, with the exception of the N-terminal residues $1-9$ and the C-terminal residues 125-127. These are highly disordered and lie in regions not defined in the electron-density maps.

The final crystallographic $R$ value for the TTR Y78F model, consisting of 1857 atoms and 196 water molecules, is $19.4 \%$ (Table 1). The final model shows excellent geometry, with all residues in the allowed regions of the Ramachandran plot. Comparison of the model obtained for TTR Y78F with the model for WT TTR deposited in the PDB (Hornberg et al.,
2000; PDB entry 1f41) revealed that there are no major differences between the two structures. The overall r.m.s. deviations were calculated after superimposing both structures and the values found were 0.156 and $0.185 \AA$, respectively, for monomers $A$ and $B$.

Residue 78 of transthyretin is located in the single $\alpha$-helix in each subunit of the homotetrameric protein (Fig. 1) and in the native TTR protein Asp18 of the $A B$ loop establishes an important hydrogen bond to the $\mathrm{OH}$ group of Tyr78, ensuring the association of the loop with the body of the molecule (Fig. 2). Thus, the tyrosine to phenylalanine exchange impairs relevant contacts between the $\alpha$-helix and the $A B$ loop, which is an interface region between the two dimers in the TTR tetramer.

The major differences between the WT and the variant protein are observed in the $F G$ loop, which also differs between monomers $A$ and $B$. This region has been reported to consistently differ between amyloidogenic variants; however, it shows good similarity between non-amyloidogenic mutants and native TTR (Hamilton et al., 1996). Nonetheless, it should be noticed that rather poor electron density has been observed in this region of the protein for a number of transthyretin structures determined so far. Subtle differences between WT and Y78F TTR are also observed for the main-chain $\mathrm{C}$ atoms belonging to the $\alpha$-helix, mainly those located in the vicinity of the mutated residue. This is explained by the differences in the contacts that residue 78 establishes in the native and in the variant protein. Whereas in the former the tyrosine is hydrogen bonded to Asp18 and, through water bridges, to the main-chain carbonyl O atoms of Pro24 and Ile26, in the Y78F variant the new residue does not allow these contacts to be established (Fig. 2). Instead, it is slightly tilted towards the indole rings of the neighbouring tryptophan residue and the $\alpha$-helical segment is not so tightly bonded to the core of the molecule. A significant shift in the $A B$ loop of the mutant protein would be predicted, which is not observed in the crystallographic structure. This can be explained by the presence of a water molecule that occupies the cavity left by the removal of the $\mathrm{OH}$ group of Tyr78; this favours efficient hydrogen bonding to Asp18 and thus stabilizes the loop (Fig. 2).

Although the helix is not displaced in the structure, the lack of hydrogen bonds may facilitate a putative displacement with consequent exposure of two highly hydrophobic residues, Phe78 and Trp79, to the solvent. The missing contact might favour the formation of a modified structure, highly prone to polymerization, after dissociation of the TTR tetramer. The significance of this contact is further corroborated by the fact that other mutations in the $\alpha$-helix have been found to increase the predisposition of TTR to amyloid formation (Hamilton et al., 1996) and it also has been argued that this is an important site for the initial folding process of TTR (Wilce et al., 1999).

Recent work has emphasized the important role that nonnative TTR monomeric species formed upon TTR dissociation may play in the fibrillogenesis cascade (Jiang et al., 2001; Quintas et al., 2001; Yang et al., 2003). 
Table 2

Principal interactions of His104 in R104H TTR.

\begin{tabular}{lll}
\hline Monomer $A$ & Side chain-main chain & His104 $\mathrm{N}^{\delta 1} \ldots$ Cys10 CO \\
& Side chain-side chain & His104 $\mathrm{N}^{\varepsilon 2} \ldots$ Glu61 O \\
Monomer $B$ & Side chain-main chain & His104 $\mathrm{N}^{\delta 1} \ldots$ Cys10 CO \\
& & His104 $\mathrm{N}^{\varepsilon 2} \ldots$ Thr123 O \\
\hline
\end{tabular}

It seems that the displacement of the $\alpha$-helix might be the structural feature in TTR Y78F that leads to the partially unfolded monomers that are the building blocks of the TTR amyloid fibrils. Interestingly, results from molecular-dynamics simulations in TTR monomers showed that the $\alpha$-helix in L55P and V30M TTR suffers large r.m.s. fluctuations (Yang et al., 2003).

The reported interaction of the tetrameric soluble form of this variant protein with a monoclonal antibody raised against highly amyloidogenic mutants and amyloid fibrils (Goldsteins et al., 1999) remains unclear since it is not known which region of TTR is recognized by the antibody.

\section{TTR R104H}

TTR R104H is a transthyretin mutation that has been described as capable of exerting a protective effect against the development of the disease in compound heterozygotic carriers of the V30M mutation (Terazaki et al., 1999).

Crystals of the recombinant TTR R104H belong to space group $P 2{ }_{1} 2{ }_{1}$, as is the case for WT TTR, with two monomers in the asymmetric unit. The final refined model, which has an $R$ value of $19.9 \%$, comprises 1841 atoms and 187 water molecules. The $\mathrm{N}$ - and C-terminal residues (1-9 and 126-127) are not defined in the electron-density maps and were thus excluded from the final model. The final model has excellent geometry, with no residues in disallowed regions of the Ramachandran plot; $90.3 \%$ of amino acids lie in the core region and the remainder in the allowed region.

Again, only subtle differences could be observed between the structures of TTR R104H and WT TTR. Analysis at the dimer-dimer and monomer-monomer interfaces showed no relevant differences from the homologous interaction surfaces in WT TTR. The dissimilarities are also negligible when TTR R104H is compared with TTR T119M, another TTR mutation with protective effects on the development of FAP in carriers of TTR V30M. Residue 104 belongs to the $F G$ loop, which is located on the surface of the transthyretin monomer. This is a region that has been claimed to be involved in amyloid formation, as discrepancies have been observed between several amyloidogenic variants and WT TTR and the nonamyloidogenic A109T variant protein (Hamilton et al., 1996). The main-chain $\mathrm{C}$ atoms of the residues belonging to the $F G$ loop in TTR R104H clearly deviate from the positions adopted in the native TTR structure. However, the contribution of this shift to the non-amyloidogenic behaviour of the protein is difficult to interpret since, as previously mentioned, this is a region where the electron density is often poorly defined and the different $\mathrm{C}^{\alpha}$ traces that have been observed may be a result of the protein flexibility in this region rather than a consistent amyloidogenic feature.

While Arg104 is not well defined in WT TTR, the new side chain of the mutated residue, His104, presents different conformations in the two monomers (Table 2 and Fig. 3). In monomer $A$ it is buried and stacked between Pro11 and Pro125, thus contributing to the stabilization of the C- and $\mathrm{N}$-terminal regions. In monomer $B$ the imidazole ring is hydrogen bonded to the Cys10 main-chain carbonyl. This stabilization effect introduced by the mutation is expected to prevent conformational changes that lead to fibril formation, indicating that the $\mathrm{N}$-terminal region at the beginning of strand $A$ might be implicated in this process. Recent studies on the formation and structure of TTR amyloid fibrils have suggested that the strands $C$ and $D$ undergo a shift from their native positions and become structurally less defined, leaving strands $A$ and $B$ exposed to the solvent (Gales et al., 2003; Olofsson et al., 2004). Intermonomeric association via strands $A / A^{\prime}$ and $B / B^{\prime}$ would drive the formation of a continuous $\beta$-sheet, providing solvent protection to the otherwise exposed novel edge region (Serag et al., 2002). In the R104H variant, the new hydrogen bond between His104 and the Cys10 mainchain carbonyl could prevent the hypothesized novel contacts being established by strand $A$. Characterization of TTR mutants from human serum has revealed extensive posttranslational modifications of the Cys10 residue (Theberge et al., 1999). Of particular importance may be S-sulfonation, which may interact with other regions of the protein through its charged nature. The above-mentioned interaction observed in the TTR R104H protein may hinder access to the side-chain thiol of Cys10 and might thereby prevent generation of some or all of the post-translational modifications at Cys10 that may be implicated in amyloidogenesis. As is the case for TTR T119M, the new variant shows an overall increase in the protein stability as a result of the new contacts. The mutation in TTR T119M occurs in the T4-binding channel, whereas in the new TTR R104H variant the mutated residue is located at the protein surface and far from the interaction region. This is in agreement with the observed differences in affinity for T4 of the two variants (Almeida et al., 2000). Though both TTR T119M and TTR R104H show an increased T4-binding affinity when compared with native TTR, this increase is much larger for TTR T119M, in which closer contacts are established between the protein and the hormone (Sebastião et al., 2001). Structural information on the TTR R104H-T4 complex as well as on TTR V30M/R104H is of importance for a more detailed characterization of the features responsible for the beneficial effects introduced by the mutation on carriers of the amyloidogenic TTR V30M variant.

\section{Comparison of TTR Y78F and TTR R104H models}

Since only very small differences were found between the WT protein and each variant, we compared the final refined models obtained for TTR Y78F and TTR R104H, which present opposite behaviour in relation to amyloidogenesis. After superimposing both structures, the r.m.s. deviations for 
the $\mathrm{C}^{\alpha}$ of each pair of monomers were calculated (Fig. 4). The highest consistent discrepancies were observed for residues $80-85$ in the $\alpha$-helix and its vicinity. Comparison of the $B$ monomers shows another region near residue 100 where a high dissimilarity is observed. However, this corresponds to residues in the $F G$ loop, which, as previously stated, is a highly flexible region of the protein. Therefore, these results are in good agreement with the previously discussed differences between the wild-type protein and the TTR Y78F variant. Interestingly, the r.m.s. deviations for the $\mathrm{C}^{\alpha}$ atoms when monomers of TTR Y78F and TTR T119M are compared show exactly the same pattern (results not shown). These observations give further grounds to the hypothesis that a looser displacement-prone $\alpha$-helix may be the principal culprit for the strong amyloidogenic nature of the TTR Y78F variant. In the case of the R104H TTR, there are no structural displacements in the main-chain region where the mutated residue is located. It seems that the non-amyloidogenic/ protective behaviour of this variant is related to a stabilizing effect arising from the novel contacts introduced by the mutation rather than to a conformational rearrangement leading to a new non-amyloidogenic fold. The strengthening of contacts in critical regions of the protein possibly prevents key structural rearrangements necessary for the fibrillization to occur. On the other hand, the amyloidogenicity of some TTR variants may be related to structural features that help in driving onward the critical steps of the fibrillogenesis cascade.

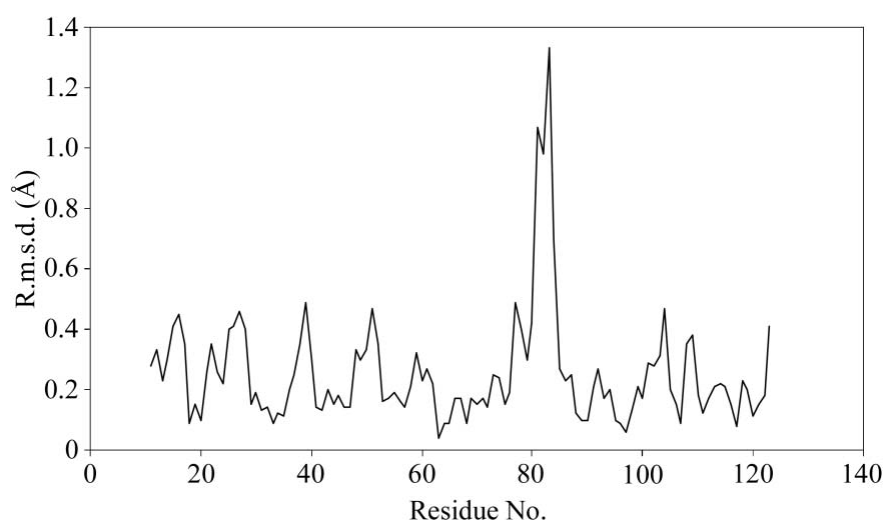

(a)

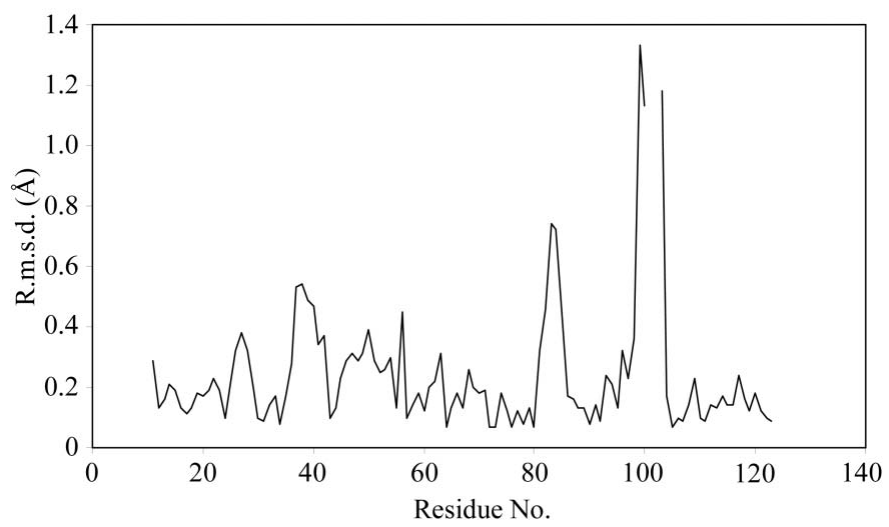

(b)

Figure 4

Plot of the differences in $\mathrm{C}^{\alpha}$ positions for residues 11-123 between the Y78F and R104H TTR variants for monomers $A(a)$ and $B(b)$.

\section{Conclusions}

The exact mechanisms underlying protein deposition in TTRrelated amyloidosis still remain to be elucidated. Nevertheless, a comparative analysis at the molecular level of the native fold and abnormal conformations of different TTR variants has proven to be a valuable tool to characterize the fibrillogenesis cascade.

Here, we have elucidated the structures of two important TTR mutants: TTR Y78F and TTR R104H. While the former strongly suggests a relevant role for the $\alpha$-helix in the overall stability of TTR, the latter showed that N-terminal stabilization might be the key determinant of its protective effects. These results are in good agreement with other insightful data regarding fibril formation obtained not only by X-ray crystallography but also by various spectroscopy methods (Gales et al., 2003; Olofsson et al., 2004; Serag et al., 2002).

Hence, it is of great importance to acquire structural data concerning other important TTR variants. This information may give us fruitful insights into other important steps of the complex fibrillogenesis cascade and thus provide us with novel targets for therapeutic intervention.

This work was funded by grants POCTI 71999/NSE/35735 and POCTI/NSE/44821/2002 from FCT (Fundação para a Ciência e Tecnologia). We thank the excellent technical support of Paul Moreira in the preparation of recombinant proteins. We acknowledge the use of beamline ID14-1 of the European Synchrotron Radiation Facility (ESRF) and we are also grateful for the technical assistance from the ESRF staff.

\section{References}

Almeida, M. R., Alves, I. L., Terazaki, H., Ando, Y. \& Saraiva, M. J. (2000). Biochem. Biophys. Res. Commun. 270, 1024-1028.

Anesi, E., Palladini, G., Perfetti, V., Arbustini, E., Obici, L. \& Merlini, G. (2001). Am. J. Med. 111, 243-244.

Blake, C. C., Geisow, M. J., Oatley, S. J., Rerat, B. \& Rerat, C. (1978). J. Mol. Biol. 121, 339-356.

Brünger, A. T., Adams, P. D., Clore, G. M., DeLano, W. L., Gros, P., Grosse-Kunstleve, R. W., Jiang, J.-S., Kuszewski, J., Nilges, M., Pannu, N. S., Read, R. J., Rice, L. M., Simonson, T. \& Warren, G. L. (1998). Acta Cryst. D54, 905-921.

Collaborative Computational Project, Number 4 (1994). Acta Cryst. D50, 760-763.

Eneqvist, T., Andersson, K., Olofsson, A., Lundgren, E. \& SauerEriksson, A. E. (2000). Mol. Cell, 6, 1207-1218.

Gales, L., Cardoso, I., Fayard, B., Quintanilha, A., Saraiva, M. J. \& Damas, A. M. (2003). J. Biol. Chem. 278, 11654-11660.

Goldsteins, G., Persson, H., Andersson, K., Olofsson, A., Dacklin, I., Edvinsson, A., Saraiva, M. J. \& Lundgren, E. (1999). Proc. Natl Acad. Sci. USA, 96, 3108-3113.

Hamilton, J. A., Steinrauf, L. K., Braden, B. C., Murrell, J. R. \& Benson, M. D. (1996). Amyloid, 3, 1-12.

Hooft, R. W., Vriend, G., Sander, C. \& Abola, E. E. (1996). Nature (London), 381, 272.

Hornberg, A., Eneqvist, T., Olofsson, A., Lundgren, E. \& SauerEriksson, A. E. (2000). J. Mol. Biol. 302, 649-669.

Jacobson, D. R., McFarlin, D. E., Kane, I. \& Buxbaum, J. N. (1992). Hum. Genet. 89, 353-356. 
Jiang, X., Smith, C. S., Petrassi, H. M., Hammarstrom, P., White, J. T., Sacchettini, J. C. \& Kelly, J. W. (2001). Biochemistry, 40, 1144211452.

Laskowski, R., MacArthur, M., Hutchinson, E. \& Thornton, J. (1993). J. Appl. Cryst. 26, 283-291.

Leslie, A. G. W. (1992). In Crystallographic Computing 5: From Chemistry to Biology, edited by D. Moras, A. D. Podjarny \& J. C. Thierry. Oxford University Press.

Magy, N., Liepnieks, J. J., Gil, H., Kantelip, B., Dupond, J. L., KluveBeckerman, B. \& Benson, M. D. (2003). Amyloid, 10, 29-33.

Monaco, H. L., Rizzi, M. \& Coda, A. (1995). Science, 268, 1039 1041.

Navaza, J. (1994). Acta Cryst. A50, 157-163.

Olofsson, A., Ippel, J. H., Wijmenga, S. S., Lundgren, E. \& Ohman, A. (2004). J. Biol. Chem. 279, 5699-5707.

Quintas, A., Vaz, D. C., Cardoso, I., Saraiva, M. J. \& Brito, R. M. (2001). J. Biol. Chem. 276, 27207-27213.

Redondo, C., Damas, A. M., Olofsson, A., Lundgren, E. \& Saraiva, M. J. (2000). J. Mol. Biol. 304, 461-470.

Roussel, A. \& Cambillau, C. (1989). In Silicon Graphics Partners Directory, edited by Silicon Graphics. Mountain View, CA, USA: Silicon Graphics.
Saraiva, M. J. (1996). J. Peripher. Nerv. Syst. 1, 179-188.

Saraiva, M. J. (2001). Hum. Mutat. 17, 493-503.

Sebastião, M. P., Lamzin, V., Saraiva, M. J. \& Damas, A. M. (2001). J. Mol. Biol. 306, 733-744.

Sebastião, M. P., Saraiva, M. J. \& Damas, A. M. (1998). J. Biol. Chem. 273, 24715-24722.

Serag, A. A., Altenbach, C., Gingery, M., Hubbell, W. L. \& Yeates, T. O. (2002). Nature Struct. Biol. 9, 734-739.

Sheldrick, G. M. \& Schneider, T. R. (1997). Methods Enzymol. 277, 319-343.

Steinrauf, L. K., Hamilton, J. A., Braden, B. C., Murrell, J. R. \& Benson, M. D. (1993). J. Biol. Chem. 268, 2425-2430.

Terazaki, H., Ando, Y., Misumi, S., Nakamura, M., Ando, E., Matsunaga, N., Shoji, S., Okuyama, M., Ideta, H., Nakagawa, K., Ishizaki, T., Ando, M. \& Saraiva, M. J. (1999). Biochem. Biophys. Res. Commun. 264, 365-370.

Theberge, R., Connors, L., Skinner, M., Skare, J. \& Costello, C. E. (1999). Anal. Chem. 71, 452-459.

Wilce, J. A., Salvatore, D., Wade, J. D. \& Craik, D. J. (1999). Eur. J. Biochem. 262, 586-594.

Wojtczak, A. (1996). Acta Cryst. D52, 758-765.

Yang, M., Lei, M. \& Huo, S. (2003). Protein Sci. 12, 1222-1231. 